\title{
Radiographic and Clinical Evolution of the Oxford Unicompartmental Knee Arthroplasty
}

\author{
Riccardo D’Ambrosi, MD ${ }^{1}$ Manuel J. de S.V. da Silva, MD ${ }^{2}$ João L. M. Moura, MD ${ }^{3}$ Ilaria Mariani, MSc ${ }^{4}$ \\ Luca D. Serrao, MD ${ }^{1}$ Fabrizio Di Feo, MD ${ }^{1}$ Nicola Ursino, MD ${ }^{1}$ \\ ${ }_{1}^{1}$ IRCCS Istituto Ortopedico Galeazzi, Milan, Italy \\ 2 Trofa Saúde Hospital Braga Centro, Braga, Portugal; ICVS/3B’s, PT \\ Government Associated Laboratory, Braga/Guimarães, Portugal; \\ School of Medicine, Minho University, Braga, Portugal; Clinical \\ Academic Center-Braga (2CA-Braga), Braga, Portugal \\ ${ }^{3}$ Senhora da Oliveira Hospital, Guimarães, Portugal; Trofa Saúde \\ Hospital Braga Centro, Braga, Portugal \\ ${ }^{4}$ Institute for Maternal and Child Health, IRCCS "Burlo Garofolo", \\ Trieste, Italy \\ J Knee Surg 2023;36:246-253. \\ Address for correspondence Riccardo D'Ambrosi, MD, IRCCS Istituto \\ Ortopedico Galeazzi, Via Galeazzi 4, Milan 20161, Italy \\ (e-mail: riccardo.dambrosi@hotmail.it).
}

\begin{abstract}
Keywords

- unicompartmental knee arthroplasty

- Oxford

- mobile bearing

- tibial slope

- implant positioning

The aim of the study is to evaluate whether the use of the new instrumentation Microplasty (MP) improves component positioning and the reliability of the surgical technique, reducing the implant outliers from the recommended range and providing a more accurate resection, while avoiding insufficient or excessive tibial resection and clinical scores. We prospectively analyzed clinical and radiographic outcomes of three consecutive cohorts for a total of 227 implants at a minimum follow-up of 36 months. The first cohort consisted of 67 Oxford unicompartmental knee arthroplasty (OUKA), using the phase III (Ph-III). The second cohort consisted of 136 OUKA, with the MP instrumentation. The third cohort consisted of 24 hypoallergenic OUKA, using the MP instrumentation (TiNbN). Postoperative alignment of the knee in the coronal and sagittal plane was measured using radiographs. No clinical differences were found among the three groups $(p>0.05)$. A significant difference was found on the slope between Ph-III and MP $(p=0.0005)$. Moreover, a significant difference was found in tibial angle and in tibial slope in arthroplasty with femoral size small (S), compared with size medium (M) or large (Ly) (tibia varus/valugs angle: $p=0.0484$; tibial slope: $p=0.04)$. Similar results were found between small $(A A, A, B)$ tibial size and large $(C, D, E, F)$ tibial size for tibial varus/valgus $(p=0.03)$ angle and tibial slope $(p=0.003)$. A significant difference was found between Ph-III and MP in tibial slope in patients with body mass index $(\mathrm{BMI}) \geq 25 \mathrm{~kg} / \mathrm{m}^{2}(p=0.0003)$. A positive correlation was noted between the femoral and tibial sizes and the tibial angle and the slope, and a negative correlation between weight and the tibial slope; furthermore, a positive correlation was found between Oxford knee score and radiographic angles. The MP instrumentation seems to be effective in determining the tibial cut and, particularly, improving the tibial slope, compared with Ph-III. The tibial slope is directly affected by the weight and measurements of the components, regardless of the instruments or the number of pegs, while clinical outcomes are correlated with implant position. This prospective comparative study reflects level of evidence II.
\end{abstract}

received

July 15,2020

accepted after revision

May 31, 2021

article published online

July 16, 2021 (c) 2021. Thieme. All rights reserved.

Thieme Medical Publishers, Inc., 333 Seventh Avenue, 18th Floor, New York, NY 10001, USA
DOI https://doi.org/ 10.1055/s-0041-1731718. ISSN $1538-8506$. 
Unicompartmental knee arthroplasty (UKA) is an effective treatment for medial osteoarthritis (OA) of the knee, providing a greater range of motion with more natural knee kinematics, lower rate of complication, and a high satisfaction, compared with total knee arthroplasty (TKA). ${ }^{1}$ The Oxford UKA (OUKA) is one of the most-used mobile-bearing implants which was developed to reduce the wear of the implant and increase the contact areas between the components during all phases of the range of motion. ${ }^{2,3}$ The OUKA Phase III (Ph-III; Zimmer Biomet, Warsaw, IN) demonstrates excellent results, providing long-term survivorship up to $91 \%$ for 20 years in some studies published by the developers of the implant, ${ }^{4}$ but the national registries show a higher revision rate due to loosening or bearing dislocation. Ph-III was introduced in 1998, specifically for medial unicompartmental use, with a minimally invasive approach. ${ }^{5}$ The single size of the femoral component (used in all the Ph-I and -II implants) was replaced by five parametric sizes, the universal tibial plateau was replaced by right- and left-handed tibial components, and the bearings were modified to diminish the likelihood of impingement and rotation. The instruments were miniaturized to facilitate their use through a small parapatellar arthrotomy. $^{5}$

The minimally invasive technique may cause a lower intraoperative visualization of the bone landmarks, causing difficult positioning of the components, especially in lowvolume surgeons. ${ }^{6}$ The most frequent cause of mistakes was the excess of tibial resections, using 5- or 6-mm insert and the wrong positioning of the femoral component, especially in the sagittal plane, due to the variability of the insertion of the intramedullary rod. ${ }^{7}$ The new Microplasty (MP) instrumentation was developed to overcome these problems and to improve the reproducibility of the procedure, providing better ligament balancing with adequate bone resection and correct alignment of the implants. The instruments were introduced in 2012, with the aim of making the operation more reliable, achieving a correct tibial resection height, facilizing the positioning of the femoral component, and having a system for preventing impingement. ${ }^{8}$

Moreover, due to the high prevalence of cutaneous metal hypersensitivity, ranging from 10 to $48 \%$ in the general population, an anallergic model of OUKA has been developed with a titanium nobium nitride (TiNbN) coating, using the MP instrumentation but with only one femoral peg. 9,10

The aim of the study is to evaluate whether the use of the new instrumentation MP improves component positioning and the reliability of the surgical technique, reducing the implant outliers from the recommended range and providing a more accurate resection, while avoiding insufficient or excessive tibial resection and clinical scores. The secondary purpose is to evaluate whether a single femoral peg affects the final positioning of the implant.

\section{Materials and Methods}

The study was conducted following the STROBE checklist for case-control study. ${ }^{11}$ Written, informed consent was obtained from all patients. Data are prospectively collected at our institution from all patients undergoing knee arthroplasty with the approval of the institutional review board.

We prospectively analyzed the radiographs and records of three consecutive cohorts of a total of 227 implants. The first cohort consisted of 67 consecutive minimally invasive implantations of the OUKA, using Ph-III. The second cohort consisted of 136 consecutive series of minimally invasive OUKA with the Oxford MP instrumentation. The third cohort consisted of 24 consecutive minimally invasive hypoallergenic OUKA, with a single femoral peg, using the MP instrumentation.

In all patients, surgery was performed by the senior author, experienced in UKA arthroplasty between May 2012 and November 2017. ${ }^{12}$

In all three groups, surgery was performed for isolated anteromedial OA or avascular necrosis (AVN) of the medial femoral condyle with bone on bone. Preoperative magnetic resonance imaging (MRI) confirmed that cruciate and collateral ligaments were functionally intact in all the patients. Radiographic inclusion criteria followed those defined by the Oxford group. ${ }^{13}$ Exclusion criteria for participation in this study were missing data or X-rays which were not suitable for an exact measurement, revision surgery, and previous surgery of the affected knee (except for arthroscopy for meniscectomy).

\section{Surgical Technique}

\section{Oxford Phase III}

A conventional technique was used as described by the manufacturer (Zimmer Biomet, Warsaw, IN). The level of tibial resection was estimated, so that the saw cut passed 2- or 3-mm below the deepest part of the erosion. The intramedullary rod was inserted into the femur through a hole $1-\mathrm{cm}$ anterior to the anteromedial corner of the intercondylar notch. The femoral drill guide was aligned visually parallel to the long axis of the tibia, in the middle of the condyle, and parallel to the intramedullary femoral rod in the coronal and sagittal planes. ${ }^{14}$

\section{Oxford Microplasty}

According to the manufacturer, there are essential differences between the conventional technique and the operation technique using the MP instrumentation. These differences relate to the level of resection of the tibia and the positioning of the femoral component. The first improvement of the updated instruments is the use of femoral sizing spoons (1$3 \mathrm{~mm}$ ) which are placed around the central femoral condyle to tension the medial collateral ligament back to normal. After applying the tibial saw guide parallel with the long axis of the tibia in both the coronal and sagittal planes, the sizing spoon is connected to the saw guide with a G-clamp (3 or $4 \mathrm{~mm}$ ), depending on the expected thickness of the mobile bearing. The second feature addresses the femoral preparation. The intramedullary rod is placed as with Ph-III instrumentation and linked to the femoral drill guide with a linking bar, ensuring an alignment of the femoral component of 7 degrees in the coronal and 10 degrees in the sagittal plane in relation to the intramedullary rod. ${ }^{15}$ 


\section{Cementation Procedure}

Sclerotic surfaces are perforated with a drill bit and then cleaned with pulsatile lavage and dried. We utilize a cement gun and osteotome to pressurize the cement into the drill holes and tibial keel slot. Refobacin Bone Cement R (Zimmer Biomet, Warsaw, IN) is the cement normally used for this procedure. The tibia is cemented first and excess cement is removed with the Woodson elevators, nerve hooks, and small suction tips. The femoral implant is then cemented, excess cement removed, and then both components pressurized at 45 degrees with a calibrated feeler gauge, until cement is hard. A feeler gauge 1-mm larger than the desired final bearing helps assure cement pressurization and penetration.

\section{Radiographic Assessment}

Positioning of the arthroplasty implants was evaluated at a minimum 36-month follow-up after surgery, as specified by radiological analysis in the Oxford Partial Knee Surgical Technique operating manual, using standing anteroposterior (AP) and lateral plain radiographs of the knee ( - Fig. $\mathbf{1}$ ).

The following parameters were measured according to the manufacturer's manuals. ${ }^{16}$

- Femoral component varus/valgus: angle between the femoral component and the femoral axis in the coronal plane. An angle of 7 degrees was seen as neutral with a range of tolerance of \pm 10 degrees.

- Tibial component varus/valgus: Angle between the tibial axis and a line drawn along the tibial tray in the coronal plane. Range of tolerance was $0 \pm 5$ degrees.

- Anteroposterior slope: angle between a line drawn along the tibial tray and perpendicular to the tibial axis in the lateral view. A slope of 7 degrees was seen as optimal with a range of tolerance of \pm 5 (2-12) degrees.

After extracting the Digital Imaging and Communications (DICOM) in Medicine data from Picture Archiving and Communications System (PACS), it was inserted into OsiriX imaging software (version 4.1.2 32-bit) and evaluated by

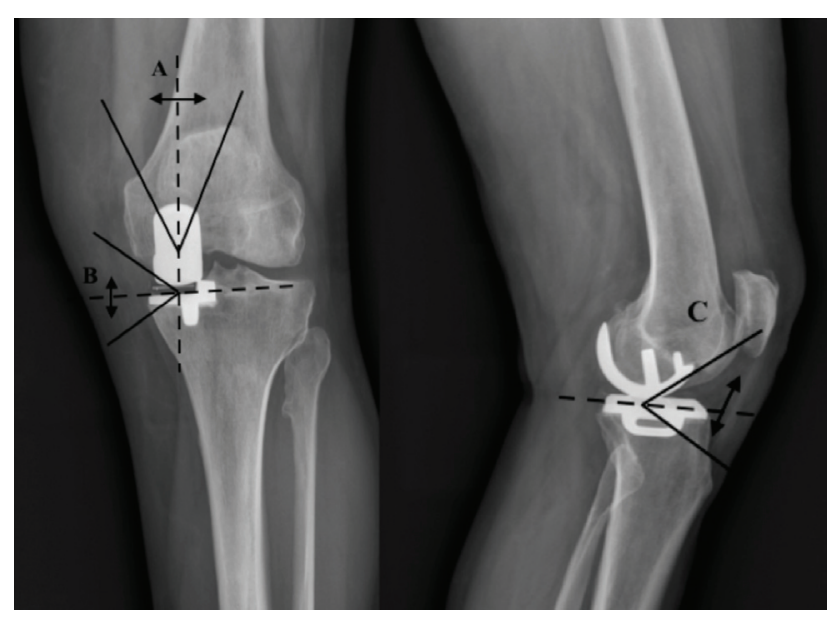

Fig. 1 Radiological parameters measured according to the manufacture manuals: femoral varus/valgus angle (A); tibial varus/valgus angle (B); anteroposterior tibial slope (C). two independent observers who were unaware of the instrumentation used for implantation. ${ }^{17}$

\section{Clinical Evaluation}

The clinical follow-up was performed by two independent clinicians who were not involved in the index surgery. The clinical evaluation consisted of evaluating each patient's Oxford knee score (OKS) at a minimum 36-month followup after surgery. ${ }^{18}$

\section{Statistical Analysis}

Assuming that the frequency of allergic patients who require UKA is between 0.10 and $0.48,{ }^{9}$ the lower conservative threshold was used in the sample size calculation. This led to a sample of 118 nonallergic patients with the same surgical technique and 12 allergic patients which was considered adequate to detect a mean slope difference of 3 degrees with a standard deviation (SD) of 3 degrees, a two-tailed type-I error of 0.05 , and a $90 \%$ power. Considering that in our setting, MP instrumentation is twice as frequent as Ph-III, 65 patients with the latter surgical technique and 13 allergic patients were needed to detect a mean slope difference of 3 , with a SD of 3, a two-tailed type-I error of 0.05 , and a $90 \%$ power. Descriptive statistical analysis, mean and $\mathrm{SD}$, (mean $\pm \mathrm{SD}$ ), and percentage for continuous and categorical variables were performed on all measures. Independence from demographic data was tested with the Chi-squared or Fisher's exact test. Femoral varus/valgus angle, tibia varus/valgus angle, anteroposterior tibial slope and OKS were tested for normality with the Shapiro-Wilk test. Based on this result, comparisons of their means within the categories of implants were tested with a one-way analysis of variance or, in the case of violation of its assumptions, with a nonparametric analysis (Kruskal-Wallis test). Bonferroni's correction was used for multiple comparisons. The $t$-test or Wilcoxon-Mann-Whitney test were performed to compare means within body mass index (BMI), age, femoral size, tibial size, and bearing size, dichotomized at their average value or at a threshold of clinical relevance. A subgroup analysis was performed with the same statistical methods to compare the mean differences in the subsets defined by implant, BMI, age, femoral size, tibial size, and bearing size. The results were expressed as mean and SD, and the $p$-value of each comparison was reported. Correlations among continuous and ordinal variables were evaluated with the Spearman's rank correlation test. A two-tailed $p$-value of less than 0.05 was considered statistically significant. Statistical analyses were performed using SAS.

\section{Results}

A total of 242 patients met inclusion and exclusion criteria, 227 of which completed the entire follow-up. Fifteen patients were excluded due to failure implants during the follow-up period. Detailed reasons of failure are reported in - Table 1. The 227 patients were included in the study with a mean age of $69.5 \pm 7.9$ years and a mean BMI of $27.8 \pm 4.2$ 
Table 1 Causes of failure after medial Oxford unicompartmental knee arthroplasty

\begin{tabular}{|l|l|}
\hline $\begin{array}{l}\text { Number of } \\
\text { patients }\end{array}$ & Failure \\
\hline 5 & Aseptic mobilization \\
\hline 4 & Bearing dislocation \\
\hline 2 & Metal hypersensitivity \\
\hline 1 & $\begin{array}{l}\text { Anterior cruciate ligament injury } \\
\text { and subsequent aseptic } \\
\text { mobilization and synovitis }\end{array}$ \\
\hline 1 & Periprosthetic tibial fracture \\
\hline 1 & Fracture and dislocation of the bearing \\
\hline 1 & Arthritis of the lateral compartment \\
\hline
\end{tabular}

$\mathrm{kg} / \mathrm{m}^{2}$. Eighty-three were males (36.6\%) and 144 females (63.4\%). Of the 227 arthroplasties, 24 (10.6\%) were anallergic (TiNbN), 67 with the Ph-III instrumentations (29.5\%), while 136 with MP with two femoral pegs (59.9\%). The mean tibia varus/valgus angle was $2.9 \pm 2.4$ degrees, and the mean femur varus/valgus angle was $7.1 \pm 5.1$ degrees, while the mean tibial slope was $5.5 \pm 3.1$ degrees. Detailed data are reported in - Table 2 .

\section{Analysis of the Angles}

Analyzing the angles between the three different groups, the only statistically significant difference concerned the tibial slope between Ph-III and MP $(p=0.0005)$. Detailed results are reported in - Table $\mathbf{3}$. Moreover, we found a significant difference in tibial angle and tibial slope in arthroplasty with small femoral size (size S), compared with sizes medium or large ( $\mathrm{M}$ or $\mathrm{L}$; tibia varus/valgus angle: $p=0.0484$; tibial slope: $p=0.04)$. Similar results were found between small (AA, A, B) tibial size and large (C, D, E, F) tibial size, regarding tibial varus/valgus $(p=0.03)$ angle and tibial slope $(p=0.003)$. Detailed results are reported in - Table 4 .

\section{Oxford Knee Score}

Analyzing the clinical scores between the three different groups, no statistical differences were found $(p>0.05)$. Detailed results are reported in -Table 3.

\section{Subgroup Analysis}

A significant difference was found between Ph-III and MP in patients with $\mathrm{BMI}<25 \mathrm{~kg} / \mathrm{m}^{2}$ in femoral component positioning ( $4.6 \pm 4.3$ vs. $8.4 \pm 5.7$ degrees; $p=0.03$ ). Another difference between the two groups was found in tibial slope, in patients with BMI $\geq 25 \mathrm{~kg} / \mathrm{m}^{2}(3.7 \pm 3.4$ vs. $6.0 \pm 3.1$ degrees; $p=0.0003$ ). Comparing the component size, a significant difference was found between Ph-III and MP in tibial slope, in patients with femoral size $S(4.3 \pm 3.6$ vs. $6.5 \pm 3.0$ degrees; $p=0.001$ ) and small tibial component (AA, A, and $\mathrm{B}$ ) $(4.4 \pm 3.7$ vs. $6.5 \pm 2.9$ degrees; $p=0.012)$. Finally, a significant difference was found in tibial slope in patients with bearing size $3 \mathrm{~mm}$ between TiNbn and Ph-III (6.4 \pm 2.8 vs. 4.0
Table 2 Demographic data of the patients included in the study

\begin{tabular}{|c|c|}
\hline Variables & $n=227$ \\
\hline Age (y) & $69.5 \pm 7.9$ \\
\hline$<70$ & $101(44.5)$ \\
\hline$\geq 70$ & $126(55.5)$ \\
\hline \multicolumn{2}{|l|}{ Gender } \\
\hline Male & $83(36.6)$ \\
\hline Female & $144(63.4)$ \\
\hline Height (m) & $1.6 \pm 0.1$ \\
\hline Weight (kg) & $73.4 \pm 13.5$ \\
\hline BMI $\left(\mathrm{kg} / \mathrm{m}^{2}\right)$ & $27.8 \pm 4.2$ \\
\hline$<25$ & $64(28.2)$ \\
\hline$\geq 25$ & $136(71.8)$ \\
\hline \multicolumn{2}{|l|}{ Implant } \\
\hline TiNbN & $24(10.6)$ \\
\hline Ph-III & $67(29.5)$ \\
\hline $\mathrm{MP}$ & $136(59.9)$ \\
\hline \multicolumn{2}{|l|}{ Tibial size } \\
\hline $\mathrm{AA}$ & $47(20.7)$ \\
\hline A & $51(22.5)$ \\
\hline$B$ & $43(18.9)$ \\
\hline$C$ & $47(20.7)$ \\
\hline $\mathrm{D}$ & $26(11.5)$ \\
\hline$E$ & $12(5.3)$ \\
\hline $\mathrm{F}$ & $1(0.4)$ \\
\hline \multicolumn{2}{|l|}{ Femoral size } \\
\hline$S$ & $137(60.3)$ \\
\hline$M$ & $74(32.6)$ \\
\hline$L$ & $16(7.1)$ \\
\hline \multicolumn{2}{|l|}{ Bearing } \\
\hline 3 & $164(72.3)$ \\
\hline 4 & $45(19.8)$ \\
\hline 5 & $14(6.2)$ \\
\hline 6 & $3(1.3)$ \\
\hline 7 & $7(0.4)$ \\
\hline \multicolumn{2}{|l|}{ Side } \\
\hline Left & $108(47.6)$ \\
\hline Right & $119(52.4)$ \\
\hline Tibia component varus/valgus (degree) & $2.9 \pm 2.4$ \\
\hline Femoral component varus/valgus (degree ) & $7.1 \pm 5.1$ \\
\hline Anteroposterior tibial slope (degree ) & $5.5 \pm 3.1$ \\
\hline Follow-up (mo) & $61.2 \pm 19.1$ \\
\hline Oxford knee score & $44.7 \pm 1.9$ \\
\hline
\end{tabular}

Abbreviations: BMI, body mass index; L, large; M, medium; MP, microplasty; Ph-III, phase III; S, small; TiNbN, hypoallergenic implant. Note: Values are presented as $n(\%)$ or mean \pm standard deviation. 
Table 3 Comparison of the angles and clinical outcomes between the three different groups

\begin{tabular}{|l|l|l|l|l|l|l|l|}
\hline & \multicolumn{2}{|l|}{ Implant (mean \pm SD) } & \multicolumn{2}{l|}{ Group comparison ( $p$-value) } \\
\hline & $\begin{array}{l}\text { TiNbN } \\
(\mathbf{n}=\mathbf{2 4})\end{array}$ & $\begin{array}{l}\text { Ph-III } \\
(\mathbf{n}=\mathbf{6 7})\end{array}$ & MP (n=136) & $\begin{array}{l}\text { TiNbN versus } \\
\text { Ph-III vs. MP }\end{array}$ & $\begin{array}{l}\text { TiNbN vs. } \\
\text { Ph-III }\end{array}$ & $\begin{array}{l}\text { Ph-III vs. } \\
\text { MP }\end{array}$ & $\begin{array}{l}\text { MP vs. } \\
\text { TINbN }\end{array}$ \\
\hline $\begin{array}{l}\text { Tibia component varus/ } \\
\text { valgus (degree) }\end{array}$ & $3.2 \pm 2.2$ & $2.4 \pm 2.5$ & $3.0 \pm 2.4$ & 0.2868 & 0.4329 & 0.9631 & 0.3238 \\
\hline $\begin{array}{l}\text { Femoral component varus/ } \\
\text { valgus (degree) }\end{array}$ & $7.3 \pm 4.8$ & $6.2 \pm 5.1$ & $7.5 \pm 5.1$ & 0.2326 & 0.6401 & 0.2070 & 0.9826 \\
\hline $\begin{array}{l}\text { Anteroposterior tibial } \\
\text { slope (degree) }\end{array}$ & $5.6 \pm 3.2$ & $4.3 \pm 3.2$ & $6.0 \pm 2.9$ & $0.0008^{\text {a }}$ & 0.2347 & $0.0005^{\text {a }}$ & 1.0000 \\
\hline Follow-up (mo) & $63.2 \pm 20.1$ & $84.5 \pm 10.0$ & $49.3 \pm 9.1$ & $<.0001^{\text {a }}$ & $<.0001^{\text {a }}$ & $<.0001^{\text {a }}$ & $0.0130^{\text {a }}$ \\
\hline Oxford knee score & $44.8 \pm 2.2$ & $45.0 \pm 1.5$ & $44.6 \pm 2.0$ & 0.5780 & 0.9616 & 0.5490 & 0.9287 \\
\hline
\end{tabular}

Abbreviations: MP, microplasty; Ph-III, phase III; S, small; SD, standard deviation; TiNbN, anallergic implant.

${ }^{a}$ Statistical significant difference.

\pm 3.4 degrees; $p=0.04)$ and between Ph-III and MP $(4.0 \pm 3.4$ vs. $6.0 \pm 3.0$ degrees; $p=0.002)$. No clinical differences were found among subgroups $(p>0.05)$. Detailed results are reported in - Supplementary Appendix 1 (available in the online version).

\section{Intergroup Analysis}

In Ph-III group, a statistically significant difference was found in femoral angle between $\mathrm{S}$ and $\mathrm{M}$ or $\mathrm{L}$ femoral sizes $(1.9 \pm 2.5$ vs. $3.1 \pm 2.5$ degrees; $p=0.03$ ) and between patients with $\mathrm{BMI}<25$ and $\geq 25 \mathrm{~kg} / \mathrm{m}^{2}$ for tibial slope ( $5.5 \pm 2.4$ vs. $3.7 \pm 3.54$ degrees; $p=0.02$ ). A difference was also found in
OKS between patients with bearing size of $3 \mathrm{~mm}$ and patients with bearing $>3 \mathrm{~mm}(45.3 \pm 1.3$ vs. $44.4 \pm 1.6 ; p=0.03)$.

In MP group, a significant difference was found in tibial slope between $S$ and $M$ or $L$ femoral sizes ( $6.5 \pm 3.0$ vs. $5.3 \pm 2.8$ degrees $p=0.01$ ) and between small (AA, A, B) and large (C, D, E, F) tibial components ( $6.5 \pm 2.9$ vs. $5.2 \pm 2.9$ degrees; $p=0.01)$. A clinical difference was found among patients with $S$ and $M$ or $L$ femoral sizes $(44.9 \pm 2.0$ vs. $44.1 \pm 1.9 ; p=0.02$ ) and patients with small (AA, A, B) and large (C, D, E, F) tibial components ( $44.9 \pm 1.9$ vs. $44.1 \pm 2.0$; $p=0.03$ ). Detailed results are reported in -Supplementary Appendix 1 (available in the online version).

Table 4 Clinical and radiographic analysis of the subgroups

\begin{tabular}{|c|c|c|c|c|c|c|c|c|c|}
\hline & \multirow[b]{2}{*}{$n$} & \multicolumn{2}{|c|}{$\begin{array}{l}\text { Tibia component } \\
\text { varus/valgus (degree) }\end{array}$} & \multicolumn{2}{|c|}{$\begin{array}{l}\text { Femoral component } \\
\text { varus/valgus (degree) }\end{array}$} & \multicolumn{2}{|c|}{$\begin{array}{l}\text { Antero posterior tibial } \\
\text { slope (degree) }\end{array}$} & \multicolumn{2}{|c|}{ Oxford knee score } \\
\hline & & Mean \pm SD & $p$-Value & Mean \pm SD & $p$-Value & Mean \pm SD & $p$-Value & Mean \pm SD & $p$-Value \\
\hline \multicolumn{10}{|l|}{$\operatorname{BMI}\left(\mathrm{kg} / \mathrm{m}^{2}\right)$} \\
\hline$<25$ & 64 & $2.7 \pm 2.6$ & 0.8515 & $7.0 \pm 5.5$ & 0.9017 & $6.0 \pm 2.4$ & 0.1551 & $44.9 \pm 1.8$ & 0.2364 \\
\hline$\geq 25$ & 163 & $3.0 \pm 2.4$ & & $7.1 \pm 5.0$ & & $5.3 \pm 3.4$ & & $44.6 \pm 1.9$ & \\
\hline \multicolumn{10}{|l|}{ Age (years) } \\
\hline$<70$ & 101 & $2.8 \pm 2.3$ & 0.4559 & $7.2 \pm 5.1$ & 0.7563 & $5.7 \pm 3.4$ & 0.3612 & $44.9 \pm 1.8$ & 0.2813 \\
\hline$\geq 70$ & 126 & $3.0 \pm 2.5$ & & $7.0 \pm 5.1$ & & $5.3 \pm 2.9$ & & $44.6 \pm 1.9$ & \\
\hline \multicolumn{10}{|l|}{ Femoral size } \\
\hline $\mathrm{S}$ & 137 & $2.6 \pm 2.4$ & 0.0484 & $7.2 \pm 5.3$ & 0.7077 & $5.9 \pm 3.4$ & 0.0465 & $44.9 \pm 1.8$ & 0.0532 \\
\hline $\mathrm{M}$ or $\mathrm{L}$ & 90 & & $3.2 \pm 2.4$ & & $6.9 \pm 4.8$ & & $5.0 \pm 2.7$ & $44.4 \pm 1.9$ & \\
\hline \multicolumn{10}{|l|}{ Tibial size } \\
\hline$A A, A, B$ & 141 & $2.6 \pm 2.5$ & $0.0327^{\mathrm{a}}$ & $7.0 \pm 5.3$ & 0.7132 & $5.9 \pm 3.2$ & $0.0033^{\mathrm{a}}$ & $44.9 \pm 1.8$ & 0.0993 \\
\hline$C, D, E, F$ & 86 & $3.3 \pm 2.2$ & & $7.3 \pm 4.7$ & & $4.8 \pm 2.8$ & & $44.4 \pm 1.9$ & \\
\hline \multicolumn{10}{|c|}{ Bearing (mm) } \\
\hline 3 & 164 & $2.9 \pm 2.3$ & 0.9509 & $7.3 \pm 5.3$ & 0.3282 & $5.5 \pm 3.2$ & 0.6292 & $44.8 \pm 1.8$ & 0.2360 \\
\hline$>3$ & 63 & $2.8 \pm 2.7$ & & $6.6 \pm 4.5$ & & $5.3 \pm 3.0$ & & $44.5 \pm 2.0$ & \\
\hline
\end{tabular}

Abbreviations: BMI, body mass index; L, large; M, medium; S, small; SD, standard deviation.




Table 5 Significative correlations

\begin{tabular}{|l|l|l|l|l|l|l|}
\hline & \multicolumn{2}{|l|}{$\begin{array}{l}\text { Tibial component } \\
\text { varus/valgus }\end{array}$} & \multicolumn{2}{l|}{$\begin{array}{l}\text { Femoral component } \\
\text { varus/valgus }\end{array}$} & \multicolumn{2}{l|}{$\begin{array}{l}\text { Anteroposterior tibial } \\
\text { slope }\end{array}$} \\
\hline & Correlation & $p$-Value & Correlation & $p$-Value & Correlation & $p$-Value \\
\hline Weight & 0.06544 & 0.3263 & -0.04986 & 0.4548 & -0.16771 & $0.0114^{\mathrm{a}}$ \\
\hline Femoral size & 0.15886 & $0.0166^{\mathrm{a}}$ & -0.06889 & 0.3014 & -0.13611 & $0.0405^{\mathrm{a}}$ \\
\hline Tibial size & 0.13038 & $0.0498^{\mathrm{a}}$ & -0.07060 & 0.2895 & -0.23089 & $0.0005^{\mathrm{a}}$ \\
\hline Female & -0.16790 & $0.0113^{\mathrm{a}}$ & 0.02231 & 0.7381 & 0.11987 & $0.0715^{\mathrm{a}}$ \\
\hline Oxford knee score & 0.02677 & 0.6883 & 0.04206 & 0.5284 & 0.09663 & 0.1467 \\
\hline
\end{tabular}

astatistical significant correlation.

\section{Correlations}

A positive correlation between the femoral and tibial sizes and the tibial angle and the slope was noted and a negative correlation between weight and the tibial slope. OKS results correlated positively with tibial angle, femoral angle, and tibial slope. Statistically significant correlations are reported in -Table 5.

\section{Discussion}

The most important finding of this study was a higher tibial slope in patients who underwent OUKA with MP instrumentation. Moreover, it was noted that femoral and tibial sizes play a key role in tibial slope inclination. Finally, a negative correlation was found between weight and tibial slope. No clinical differences between the groups were found at the final follow-up.

A similar study was performed by Walker et al, analyzing the reproducibility of implant positioning, using MP instrumentation. ${ }^{19}$ On 300 UKA, the mean bearing thickness was statistically significant and lower with use of the updated instrumentation than with the conventional instrumentation. Additionally, the results of the current study were aligned within the accepted range of tolerance in both the coronal and the sagittal planes, with the use of the updated instrumentation, compared with the conventional Ph-III instrumentation. ${ }^{19}$

According to these results, Jang et al aimed to determine whether MP instrumentation could improve postoperative implant positioning and limb alignment. ${ }^{7}$ Statistically significant differences were identified in the varus/valgus and flexion/extension angles of the femoral implant. ${ }^{7}$

In the current study, for femoral angle, a significant difference was found only between patients with a BMI $<25$ $\mathrm{kg} / \mathrm{m}^{2}$ when compared with a BMI $\geq 25 \mathrm{~kg} / \mathrm{m}^{2}$.

The Oxford Ph-III presents five different sizes of femoral component, providing more options than the previous models. Several studies suggest preoperative selection by templating the appropriate size of the femoral component. Preoperative templating is an important step that can help in the selection of implant size, position, and alignment. However, many controversies are reported in the literature. In fact, Bothra et $\mathrm{al}^{20}$ showed poor intraobserver and interobserver agreement and concluded that the present templating system lacked reliability.
Kasis et $\mathrm{al}^{21}$ revealed a poor level of interobserver reproducibility and accuracy, regardless of a high level of intraobserver reproducibility.

In 2008, Fawzy et $\mathrm{al}^{22}$ proposed an Oxford algorithm for selecting femoral component size by investigating the optimal relationship between ideal femoral component size and patient height, gender, and tibial component size. They concluded that height based on gender was a reliable method of prediction.

An article by $\mathrm{Tu}$ et $\mathrm{al}^{23}$ developed a better preoperative intraoperative measure to predict femoral component size using an intraoperative $\mathrm{C}$-arm intensifier guide (CAIG). A significant difference between templating (59\%) and CAIG (92\%) method was found in the study cohort. In the Chinese algorithm, height based on gender and tibial size both have a greater accuracy of prediction ( 88 and $70.7 \%$ ) than the Oxford algorithm (51.1 and 59.8\%). Component size distribution and optimal tibial/femoral pairing differed from those in the Oxford report.

Furthermore, is still debated whether to use an intra- or extramedullary guide for the correct positioning of the femoral component. Some authors are moving toward extramedullary alignment which is merely an indicator of frustration with the accuracy of intramedullary alignment. ${ }^{24}$ A cadaveric study demonstrated that the use of a short and long intramedullary femoral rod may result in excessive flexion alignment error of the femoral component. Understanding the extramedullary alignment possibility and experience with the visual alignment of the femoral drill guide is essential for minimizing potential errors in the alignment of the femoral component. ${ }^{25}$

In contrast to total knee arthroplasty, tibial component rotation in UKA has a wide deviation. The tibial component tends to rotate externally, due to the lack of a distinct landmark for tibial component rotation in the small operating field. ${ }^{26}$ Although the ideal tibial component rotation in UKA is well debated, the direct relationship between postoperative tibial component rotation and clinical outcomes is not clearly described.

In 2018, Kamenaga et $\mathrm{al}^{27}$ aimed to investigate whether tibial component rotation in the axial plane could affect clinical outcomes after UKA. In 50 patients who underwent OUKA, rotation angles of tibial components had significant negative correlations with the recovery of the OKS in the 2 years following surgery, demonstrating that a trend toward poor outcome was observed when the tibial component was 
placed at a higher angle of external rotation. Moreover, the same author, ${ }^{28}$ in a subsequent study, demonstrated that, in OUKA recipients, the bearing may impinge on the lateral wall of the tibial component during flexion above 60 degrees, if the tibial component is placed too medially or if it exhibits pronounced external rotation which may limit knee function improvement postoperatively.

In the present study, tibial slope is the angle that showed the most variations and differences. In fact, the Ph-III group showed a significantly lower slope than the MP group. In addition, factors, such as femoral size, tibial size, and weight, play a key role in the tibial slope. The posterior tibial slope is well known for influencing the flexion gap in TKA but it is still unknown whether a wide gap at the deep knee flexion is necessary to attain a wide flexion angle in the UKA. ${ }^{29}$

Many issues still remain regarding the natural tibial slope. In fact, as reported by Pangaud et $\mathrm{al}^{30}$ in 378 healthy participants, posterior tibial slope is significantly influenced by sex, ethnicity, and lower limb alignment, reporting an overall mean value of 6.3 degrees.

Suzuki et $\mathrm{al}^{31}$ recently revealed the effect tibial slope has on knee flexion, extension joint gap, and the postoperative range of motion in mobile-bearing UKA. The author found that the degree of the posterior tibial slope should not be so large as to avoid joint looseness throughout every knee angle. Increasing the degree of the slope had the potential of dislocating the bearing.

In previous publications on radiographic difference, only Ph-III and MP were evaluated without considering anallergic arthroplasty. A high prevalence of cutaneous metal hypersensitivity has been reported, with 10 to $48 \%$ of the general population having experienced skin hypersensitivity to metals. ${ }^{9}$ There are several studies showing a higher prevalence of a positive patch test after implantation of metallic TKA (components and metal sensitivity accounted for $1.3 \%$ of the revisions in 2012 and in 2014 ) with $1.8 \%$ of revision TKAs attributed to "metal related pathology." This number has been steadily increasing over the years. ${ }^{9}$

In the current study, no radiographic differences were found among TiNbN group and other groups, highlighting that this model can be considered safe, although only one femoral peg is present.

These results are confirmed by Walker et al ${ }^{10}$ who evaluated the clinical outcome and survival rates of UKA, using a standard $\mathrm{CoCr}$ alloy in patients reporting signs of hypersensitivity to metal. On 82 patients, at a mean follow-up of 3 years, no local or systemic symptoms of hypersensitivity to metal were observed. One patient underwent revision surgery to a bicondylar prosthesis due to a tibial periprosthetic fracture, resulting in a survival rate of $98.8 \%$. Clinical outcome was good to excellent with a mean OKS of 42.5.

\section{Limitations}

There are several limitations to our study, such as the lack of misuration of femoral and tibial axes, using long-leg X-rays that can potentially cause deviations from the true axes. Finally, we did not consider the degree of osteoporosis and femoral bowing in our patients which might affect the position of the rod.

\section{Conclusion}

The MP instrumentation seems to be effective in determining tibial cut, particularly, in improving tibial slope, compared with Ph-III. The tibial slope is directly affected by the weight and measurements of the components, regardless of the instruments or the number of pegs, while clinical outcomes are correlated with implant position.

\section{Funding}

None.

Conflict of Interest

None declared.

\section{References}

1 Liddle AD, Judge A, Pandit H, Murray DW. Adverse outcomes after total and unicompartmental knee replacement in 101,330 matched patients: a study of data from the National Joint Registry for England and Wales. Lancet 2014;384(9952):1437-1445

2 Jackson WF, Berend KR, Spruijt S. 40 years of the Oxford Knee. Bone Joint J 2016;98-B(10(suppl B):1-2

3 Johal S, Nakano N, Baxter M, Hujazi I, Pandit H, Khanduja V. Unicompartmental knee arthroplasty: the past, current controversies, and future perspectives. J Knee Surg 2018;31(10):992-998

4 Price AJ, Svard U. A second decade lifetable survival analysis of the Oxford unicompartmental knee arthroplasty. Clin Orthop Relat Res 2011;469(01):174-179

5 Lisowski LA, Meijer LI, van den Bekerom MP, Pilot P, Lisowski AE. Ten- to 15-year results of the Oxford Phase III mobile unicompartmental knee arthroplasty: a prospective study from a nondesigner group. Bone Joint J 2016;98 B(10(suppl B):41-47

6 Liddle AD, Pandit H, Judge A, Murray DW. Effect of surgical caseload on revision rate following total and unicompartmental knee replacement. J Bone Joint Surg Am 2016;98(01):1-8

7 Jang KM, Lim HC, Han SB, Jeong C, Kim SG, Bae JH. Does new instrumentation improve radiologic alignment of the Oxford medial unicompartmental knee arthroplasty? Knee 2017;24 (03):641-650

8 Koh IJ, Kim JH, Jang SW, Kim MS, Kim C, In Y. Are the Oxford medial unicompartmental knee arthroplasty new instruments reducing the bearing dislocation risk while improving components relationships? A case control study. Orthop Traumatol Surg Res 2016; 102(02):183-187

9 Bravo D, Wagner ER, Larson DR, Davis MP, Pagnano MW, Sierra RJ. No Increased risk of knee arthroplasty failure in patients with positive skin patch testing for metal hypersensitivity: a matched cohort study. J Arthroplasty 2016;31(08):1717-1721

10 Walker T, Rutkowski L, Innmann M, et al. Unicondylar knee arthroplasty using cobalt-chromium implants in patients with self-reported cutaneous metal hypersensitivity. Bone Joint J 2019;101-B(02):227-232

11 von Elm E, Altman DG, Egger M, Pocock SJ, Gøtzsche PC, Vandenbroucke JPSTROBE Initiative. The Strengthening the Reporting of Observational Studies in Epidemiology (STROBE) statement: guidelines for reporting observational studies. Lancet 2007;370 (9596):1453-1457

12 Zhang $\mathrm{Q}$ Zhang $\mathrm{Q}$ Guo $\mathrm{W}$, et al. The learning curve for minimally invasive Oxford phase 3 unicompartmental knee arthroplasty: cumulative summation test for learning curve (LC-CUSUM). J Orthop Surg Res 2014;9:81 
13 Hamilton TW, Pandit HG, Lombardi AV, et al. Radiological Decision Aid to determine suitability for medial unicompartmental knee arthroplasty: development and preliminary validation. Bone Joint J 2016;98-B(10(suppl B):3-10R1

14 Hurst JM, Berend KR, Adams JB, Lombardi AV Jr. Radiographic comparison of mobile-bearing partial knee single-peg versus twin-peg design. J Arthroplasty 2015;30(03):475-478

15 Mohammad HR, Matharu GS, Judge A, Murray DW. New surgical instrumentation reduces the revision rate of unicompartmental knee replacement: A propensity score matched comparison of 15,906 knees from the National Joint Registry. Knee 2020;27(03): 993-1002

16 Sharkey PF, Hozack WJ, Rothman RH, Shastri S, Jacoby SM. Insall award paper. Why are total knee arthroplasties failing today? Clin Orthop Relat Res 2002;(404):7-13

17 Mujika KM, Méndez JAJ, de Miguel AF. Advantages and disadvantages in image processing with free software in radiology. J Med Syst 2018;42(03):36

18 Padua R, Zanoli G, Ceccarelli E, Romanini E, Bondì R, Campi A. The Italian version of the Oxford 12-item Knee Questionnaire-crosscultural adaptation and validation. Int Orthop 2003;27(04): 214-216

19 Walker T, Heinemann P, Bruckner T, Streit MR, Kinkel S, Gotterbarm $\mathrm{T}$. The influence of different sets of surgical instrumentation in Oxford UKA on bearing size and component position. Arch Orthop Trauma Surg 2017;137(07):895-902

20 Bothra V, Lemon G, Lang D, Smith DM, Ali AM. Reliability of templating in estimating the size of uni-condylar knee arthroplasty. J Arthroplasty 2003;18(06):780-783

21 Kasis AG, Pacheco RJ, Hekal W, Farhan MJ, Smith DM, Ali AM. The precision and accuracy of templating the size of unicondylar knee arthroplasty. Knee 2004;11(05):395-398

22 Fawzy E, Pandit H, Jenkins C, Dodd CA, Murray DW. Determination of femoral component size in unicompartmental knee replacement. Knee 2008;15(05):403-406
23 Tu Y, Xue H, Cai M, Ma T, Liu X, Xia Z. Improvement of femoral component size prediction using a $\mathrm{C}$-arm intensifier guide and our established algorithm in unicompartmental knee arthroplasty: a report from a Chinese population. Knee 2014;21(02):435-438

24 Zhang $\mathrm{Q}$, Wang $\mathrm{W}$, Liu Z, et al. A novel extramedullary technique to guide femoral bone preparation in mobile unicompartmental knee arthroplasty based on tibial cut and overall alignment. J Orthop Surg Res 2020;15(01):92

25 Kort NP, van Raay JJ, Thomassen BJ. Alignment of the femoral component in a mobile-bearing unicompartmental knee arthroplasty: a study in 10 cadaver femora. Knee 2007;14(04):280-283

26 Lee SY, Chay S, Lim HC, Bae JH. Tibial component rotation during the unicompartmental knee arthroplasty: is the anterior superior iliac spine an appropriate landmark? Knee Surg Sports Traumatol Arthrosc 2017;25(12):3723-3732

27 Kamenaga T, Hiranaka T, Kikuchi K, Hida Y, Fujishiro T, Okamoto $\mathrm{K}$. Influence of tibial component rotation on short-term clinical outcomes in Oxford mobile-bearing unicompartmental knee arthroplasty. Knee 2018;25(06):1222-1230

28 Kamenaga T, Hiranaka T, Takayama K, Tsubosaka M, Kuroda R, Matsumoto T. Adequate positioning of the tibial component is key to avoiding bearing impingement in oxford unicompartmental knee arthroplasty. J Arthroplasty 2019;34(11):2606-2613

29 Kang KT, Koh YG, Son J, Kwon OR, Lee JS, Kwon SK. Influence of increased posterior tibial slope in total knee arthroplasty on knee joint biomechanics: a computational simulation study. J Arthroplasty 2018;33(02):572-579

30 Pangaud C, Laumonerie P, Dagneaux L, et al. Measurement of the posterior tibial slope depends on ethnicity, sex, and lower limb alignment: a computed tomography analysis of 378 healthy participants. Orthop J Sports Med 2020;8(01):2325967119895258

31 Suzuki T, Ryu K, Kojima K, Oikawa H, Saito S, Nagaoka M. The effect of posterior tibial slope on joint gap and range of knee motion in mobile-bearing unicompartmental knee arthroplasty. J Arthroplasty 2019;34(12):2909-2913 